\title{
A Rotating Sphere as a Possible Source of the Kerr Metric.
}

\section{P. S. FLORIDES}

School of Mathematics, Trinity College - Dublin

Dublin Institute for Advanced Studies - Dublin

(Nuovo Cimento, $13 \mathrm{~B}, 1$ (1973))

Please correct the following misprints in this paper:

Page 2: in eq. (1.1) instead of small theta $(\theta)$ read capital theta $(\Theta)$.

Page 4: in eq. (2.3) instead of $\underset{i}{\omega_{\alpha \beta^{\prime}} \omega_{j} \beta^{\prime}} u_{l}$ read $\underset{1}{\omega_{\alpha \beta^{\prime}}}{\underset{1}{\alpha \beta^{\prime}}}_{1} u_{\alpha}$ respectively.

Page 7: in eq. (2.13), 1 (one) at the beginning of this equation should read $i$.

Page 9: eq. (2.22), 5th line from top, replace

$$
\frac{m^{2}}{5 b^{2}}+\frac{5 b^{2}}{m^{2}}\left(\frac{99 b^{3}}{35 x^{3}}-\ldots\right)+\ldots \quad \text { by } \quad \frac{m b^{2}}{5 x^{3}}+\frac{m^{2}}{5 b^{2}}\left(\frac{99 b^{3}}{35 x^{3}}-\ldots\right)+\ldots
$$

Page 9: in eq. (3.1) instead of small theta $(\theta)$ read capital theta $(\Theta)$.

Page 11: eqs. (3.8): on the right-hand-side of the two equations instead of small theta $(\theta)$ read capital theta $(\Theta)$. 